\title{
The Embedded System Integrated ECG Monitoring and Low Energy Led Light Stimulation
}

\author{
Yi-Chia Shan ${ }^{1}$, Jih-Huah $\mathrm{Wu}^{2}$, Wei-Hao Chen ${ }^{2}$, Shau-Ming Tseng ${ }^{2}$, Wei Fang ${ }^{1, *}$ \\ ${ }^{1}$ Department of Bio-Industrial Mechatronics Engineering, National Taiwan University, Taiwan \\ ${ }^{2}$ Department of Biomedical Engineering, Ming Chuan University, Taiwan
}

Copyright $\odot 2016$ by authors, all rights reserved. Authors agree that this article remains permanently open access under the terms of the Creative Commons Attribution License 4.0 International License.

\begin{abstract}
Background: Nowadays people usually have higher work pressure and faster life rhythm. In the long-term stress environment, easily lead to autonomic nervous system (ANS) disorders and lead to physiological or psychological problems. To resolve the problem, we propose an embedded system which integrates ANS monitoring and low energy light stimulation. When the ANS was detected abnormal, a recommended low energy infrared light was activated to stimulate the subject to balance ANS. Materials and Methods: The experiment instruments included electrocardiogram (ECG) module, LED array and the embedded system. ECG module received ECG signal, and then transferred analog signal to digital data. The microprocessor calculated time-domain and frequency-domain data of heart rate variability (HRV), and analyzed the time-domain data, and power spectral density data. The autonomic nervous system (ANS) of the user can be monitored by this device we developed. When the calculated values are out of the criteria, the system will alert the user to turn on infrared light to stimulate and balance ANS. LED wavelength is $850 \mathrm{~nm}$, the default output power is $10 \mathrm{~mW}$, and the default repetition rate is 10 Hz. Result: Wu et al. proposed that using low level laser to stimulate on Neiguan point (PC6) was used to adjust the ANS of the night shift workers. And in other studies, Laser array and LED array were used to stimulate the palm of the subjects with open eyes in different clinical trial, the alpha powers were both increased significantly. So the combination of ANS monitoring and light therapy was useful and feasible. Besides ECG monitoring, LED irradiation and HRV calculation, the data of the embedded system can be transmitted by UART to the remote PC for storage, display and analysis. Furthermore, the embedded system can receive the setting value from PC. For example, adjusting the irradiated dosage, LED operation rate and duty cycle to find out the influence factor of the ANS. Conclusion: an embedded system with ECG monitoring and LED infrared stimulation was realized, it combined
\end{abstract}

physiological state monitoring and light stimulation, it make the ANS feedback and real time adjustment possible.

Keywords Autonomic Nervous System, ECG, HRV, Embedded System

\section{Background/ Objectives and Goals}

Physiological signal are measured to monitor the body state and the common application in medicine instrument. For example, heart rate variability in ECG, brain wave in EEG, blood pressure, breathes rate, and blood oxygen, etc. NASA and Stanford University developed Lifeguard system, which can record ECG, body temperature, breathing rate, blood pressure, and 3-axis accelerometer [1]. Intelligent textiles are a combination of textile, electronic, information of high technological products, which integrate active electrode textile can monitor physiological signal any time [2]. Heart rate variability is the differences between heartbeat cycles. It can reflect the state of the ANS. HRV can be used as a risk judgment factor of coronary artery occlusion, to predict coronary occlusion situation [3].

Low frequency stimulation is widely used in many medical fields, such as sound, magnetic field, electric field, and light which have different treatment effect [4]. Low level laser can cause physiological change. In vitro, He-Ne laser irradiate cells whose ATP were enhanced [5]. Low level laser can help to improve the peripheral never surgery [6]. Laser also can reduce the pain of chronic disease [7]. Visual stimulation with the irradiation by white LED light (1,350 lux) can improve the seasonal affective disorder (SAD) [8]. Besides, LED is also used in dermatology [9]. In animal experiments, blue LED light can accelerate wound healing of mice [10]. Wu et al. proposed stimulating Neiguan point (PC6) with low level laser was used to adjust the ANS of the night shift workers [11]. And in other studies, Laser array and LED array were used to stimulate the palm of the subjects with open eyes in different clinical trial, the 
alpha powers were both increased significantly $[12,13]$. So the combination of ANS monitoring and light treatment was useful and feasible.

The proposed system combined with physiological signal monitoring and low power photo therapy, it is suitable in home use as a medical device. The system measured ECG signal, calculating HRV, then analyzing autonomous never state. When the calculated values are out of the criteria, the system will alert the user to turn on infrared light to stimulate and balance ANS.

\section{Methods}

The system architecture is shown in Figure 1. There are three parts included, ECG module, LED array and embedded system. Computer is for remote control, data storage. The photo of the experiment system is shown in Figure 2.

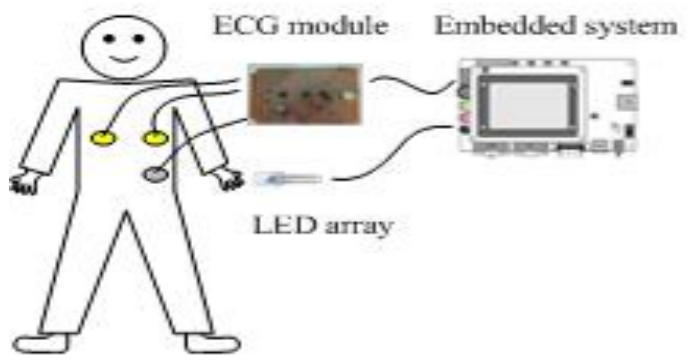

Figure 1. System architecture

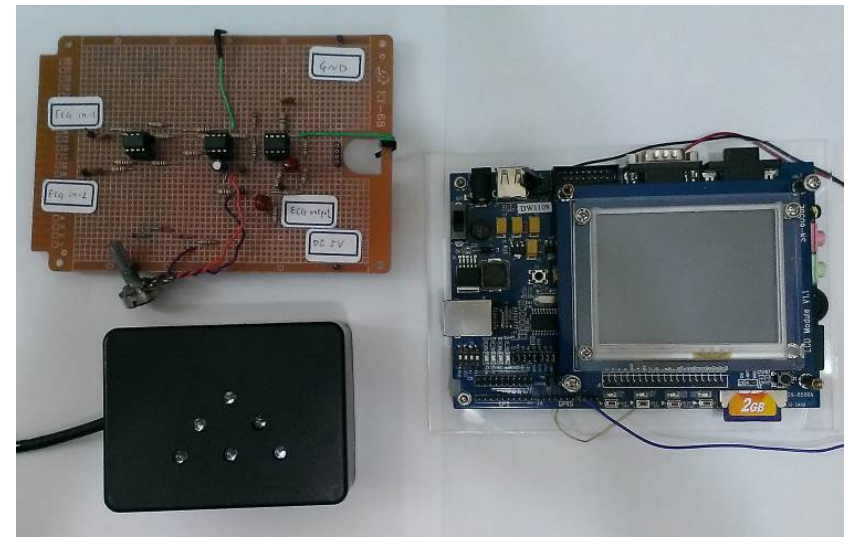

Figure 2. The ECG module, LED array, and embedded system were in the experiment system.

\subsection{ECG Module}

An electrocardiogram module is designed in this study. The scheme was from electronic laboratory guide by anonymous, and was shown in Figure 3. The ECG module includes four parts. Part (a) is an instrumentation amplifier, the gain is 21. Part (b) is an inverter operational amplifier, the gain is -100 . The total amplifier gain of ECG board is $-2,100$. Part (c) is low-pass filter with $9 \mathrm{~Hz}$ cutoff frequency, 2 poles, and Butterworth Sallen-Key configuration. Part (d) is DC level adjustment.

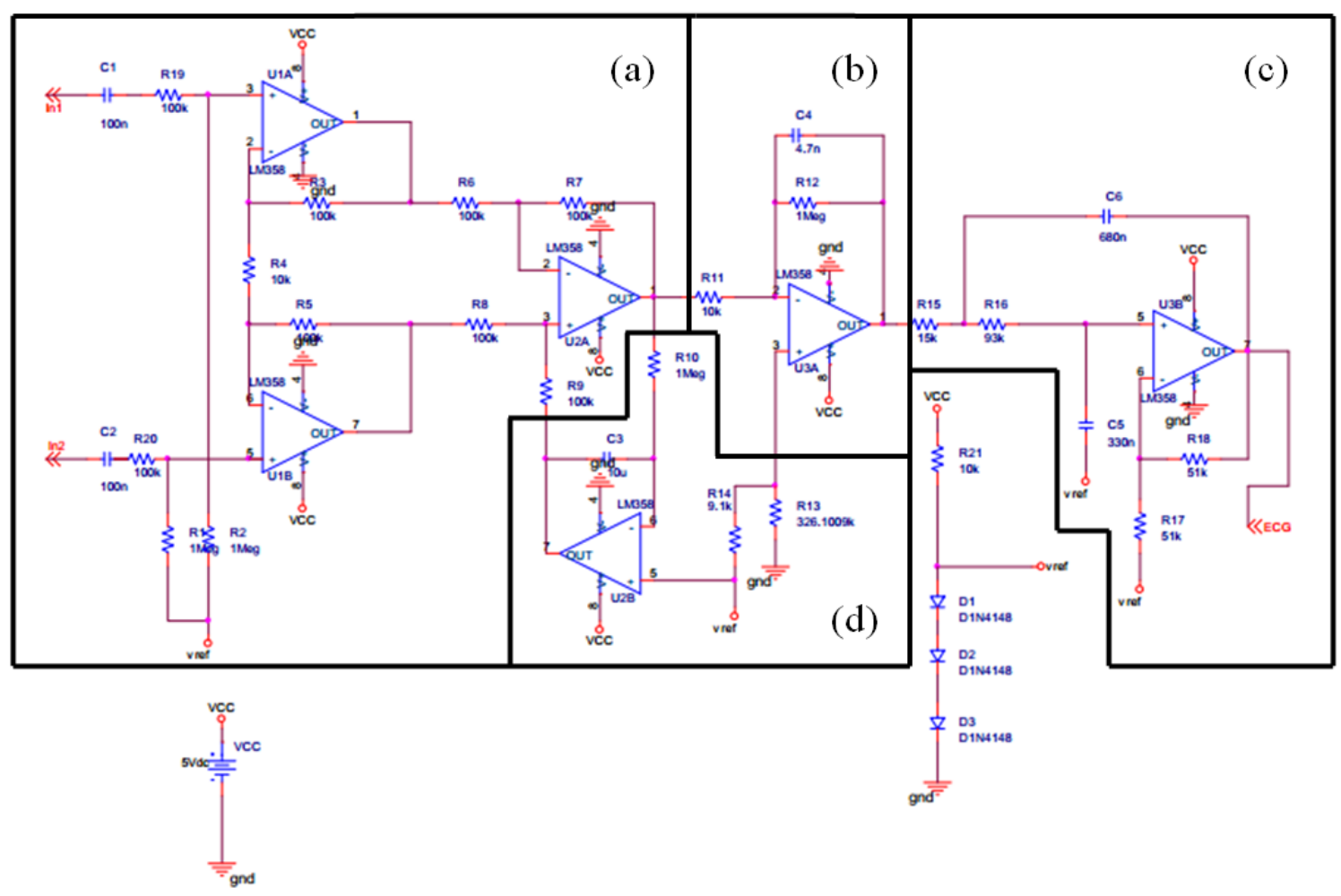

Figure 3. ECG scheme, part (a) is instrumentation amplifier, part (b) is inverter amplifier, part (c) is two order low-pass filter with $9 \mathrm{~Hz}$ cutoff frequency, part (d) is DC level adjustment. 


\subsection{LED Array Arrangement and Dose Calculation}

An array of six LED arranged in a triangle form was used to radiate near infrared light at specific body site of the subjects. More LEDs can cover wider stimulation area. For example the subject could not know Neiguan point precisely. But more LEDs can cover wider area included Neiguan point. The scheme is shown in Figure 4. LED (model no. BIR-BO13J4G, manufactured by YELLOW STONE Co., Ltd. Taiwan) was used in this study. The central wavelength of LED was $850 \mathrm{~nm}$, the irradiated angle is 20 degree, the default output power of each LED was set at $10 \mathrm{~mW}$, the operation frequency was $10 \mathrm{~Hz}$, and the duty cycle was $50 \%$. The light spot of LED can be considered as a circle. The area of the light spot was approximately equal to $28 \mathrm{~mm}^{2}$ at the skin. Thus, the dosage would be approximately $5.4 \mathrm{~J} / \mathrm{cm}^{2}$ for 5 minutes treatment. Dosage can be controlled by adjusting light duty cycle.

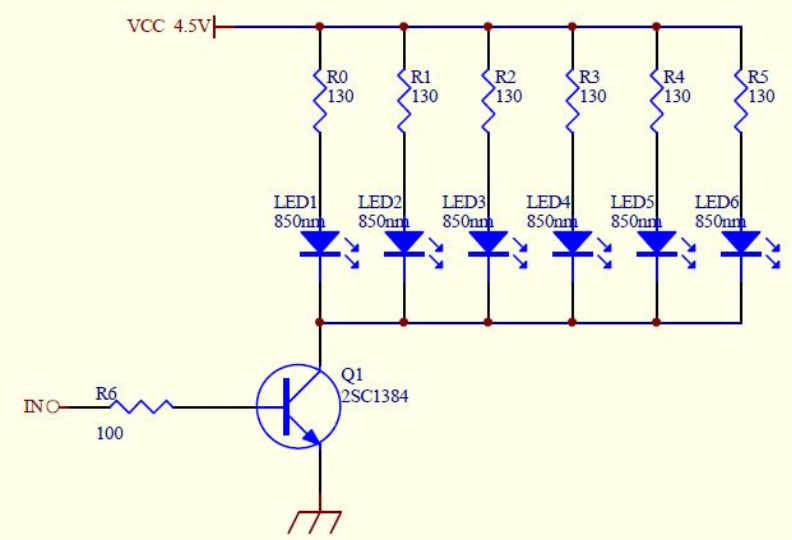

Figure 4. LED array scheme

\subsection{Embedded System}

There are processing, recording, communication and display function in the embedded system which is responsible for analyzing ECG signal and control LED irradiation. Sunplus 32-bit RISC processor was built on the development board SPCE3200. There are many interfaces included 12-bit ADC, 16-bit DAC; UART, SPI, I2C, USB, Flash, SD card; LCD; etc. in the embedded processor. The embedded system block is shown in Figure 5.

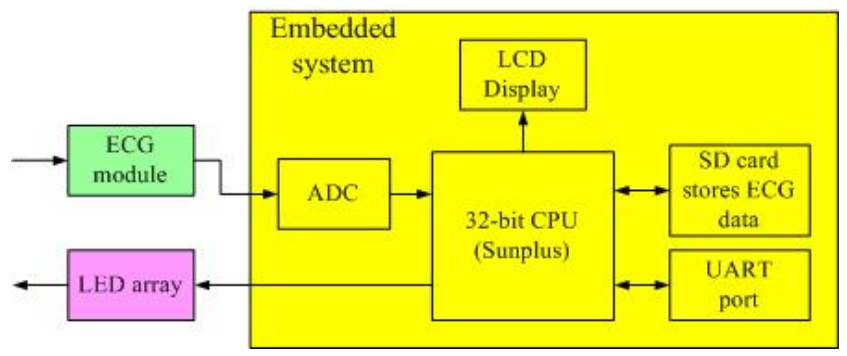

Figure 5. Embedded system block

\subsection{Experiment Procedure}

Each subject sat in a chair and was required to put LED array on Neiguan point (PC6). The subject was instructed to be relaxed, kept the eyes open and withhold from any movement. In the beginning, each subject was required to relax for five minutes in order to be in a stable physiological state. There were three sessions in the experiment procedure, before stimulation ( 5 minutes, session 1), during stimulation ( 5 minutes, session 2), and after stimulation (5 minutes, session 3). The ongoing ECG was recorded in first and third sessions.

In the experiment, $\mathrm{Ag} / \mathrm{AgCl}$ electrode pads are pasted on the chest, because the quality of ECG signal was better than the limbs. On signal electrode is pasted on the left chest, another signal electrode is on the right chest, and the ground electrode was near navel.

The recommend stimulation site was on Neiguan point (PC6). Low level light irradiation is noninvasive; the effect is moderate and less impact on user psychological pressure. The wavelength of LED light is $850 \mathrm{~nm}$ which belonged infrared light.

\subsection{Introduction of HRV Analysis}

The detection of heartbeat is from QRS complexes, and R is the peak of the complex. HRV is standard deviation of time sequence (RR-intervals). There are two types of analysis in HRV. Time domain analysis reports the activity of cardiovascular system, and frequency domain analysis reflects the sympathovagal balance of ANS.

\subsubsection{Time Domain Method}

For the time domain analysis, the RR intervals of ECG records were analyzed and the results were expressed in the form of normal-to-normal (NN) intervals. Then the standard deviation of the intervals (SDNN) was calculated.

SDNN (ms): the standard deviation of the RR intervals seen on the ECG

\subsubsection{Frequency Domain Method}

For the frequency domain analysis, Fourier transform was applied to complex heartbeat signals. The frequency analysis is provided the spectral composition of these variations. LF band $(0.04-0.15 \mathrm{~Hz})$ spectral power reflects both sympathetic and vagal influences. HF band $(0.15-0.4 \mathrm{~Hz})$ spectral power reflects vagal modulation of cardiac activity [16]. The relevant indices and signal were defined and obtained as follows:

LF: Power in low frequency range $0.04-0.15 \mathrm{~Hz}$, shown as a percentage

LF $(\%)=$ LF power / (HF power + LF power $)$

HF: Power in high frequency range $0.15-0.4 \mathrm{~Hz}$, shown as a percentage

HF $(\%)=$ HF power $/$ (HF power+ LF power)

LF: HF ratio: the ratio of LF to HF HRV 


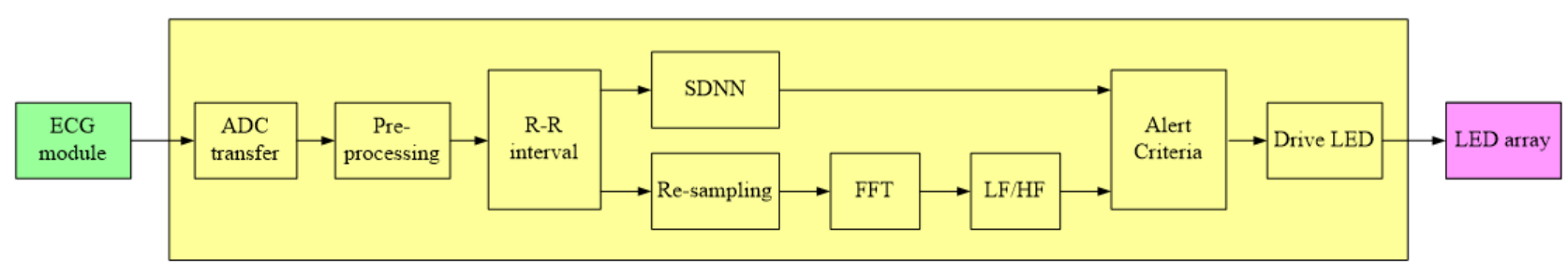

Figure 6. Function block

\subsection{Function Block}

Function block is shown in Figure 6. ECG signal was amplified and filtered by ECG module, and then the signal was transmitted to the embedded system. With internal ADC port, analog signal is converted into digital signal, and then ECG data will be processed and stored into the SD card. Then ECG signal was processed by the real-time QRS detection algorithm [14]. The pre-processed functions include differentiation, squaring and moving-window integration, and an adjustable threshold to decide the period of the heartbeat. The system will calculate HRV on time-domain and frequency-domain to analyze autonomous never state. On time domain, the heartbeat rate was calculated to get the standard deviation of normal to normal RR intervals (SDNN). On frequency domain, because the $R R$ interval of the heartbeat was not equal, firstly using Berger manner [15] to convert heartbeat as equidistant sampling, and then does the fast Fourier transform (FFT), analyze LF and HF power. When the HRV values were out of the self-defined criteria, the system will remind the subject to turn on LED stimulation to balance ANS.

\subsection{Alert Criteria}

A high risk group may be selected by the dichotomy limits of SDNN $<50 \mathrm{~ms}$ or HRV triangular index $<15$ by the guidelines of task force [16]. SDNN is related with ANS activity and LF reflects both sympathetic and vagal influences. In the study, we propose an alert concept. If SDNN or LF is out of the reference range in Table 1, and the system will remind the object to turn on LED stimulation to get ANS balance. But the complete criteria still need to be built with more reference clinical tests.

Table 1. Self-defined alert criteria

\begin{tabular}{|c|c|c|}
\hline HRV & Description & Alert range \\
\hline SDNN & $\begin{array}{c}\text { Standard deviation of all NN } \\
\text { intervals. }\end{array}$ & $<80 \mathrm{~ms}$ \\
\hline LF $(\%)$ & $\mathrm{LF} /(\mathrm{LF}+\mathrm{HF})$ & $>80 \%$ \\
\hline
\end{tabular}

\section{Results}

Figure 7 is the experiment picture of the embedded system. ECG signal is shown in the touch panel of the embedded system. In Figure 8, the white signal is QRS detection signal which is the preprocessed ECG signal after differentiating, squaring and moving-window integration [14]. Differentiating is to provide the QRS-complex slope information. Squaring is to make all data point positive and emphasize the higher frequencies. Moving-window integration is to obtain waveform feature in additional to the slope of the $\mathrm{R}$ wave. The number of samples in the width of the integration window is determined empirically. In the study, thirty two samples are averaged in moving-window integration. The red line is the adjustable threshold to decide the period of the heartbeat.

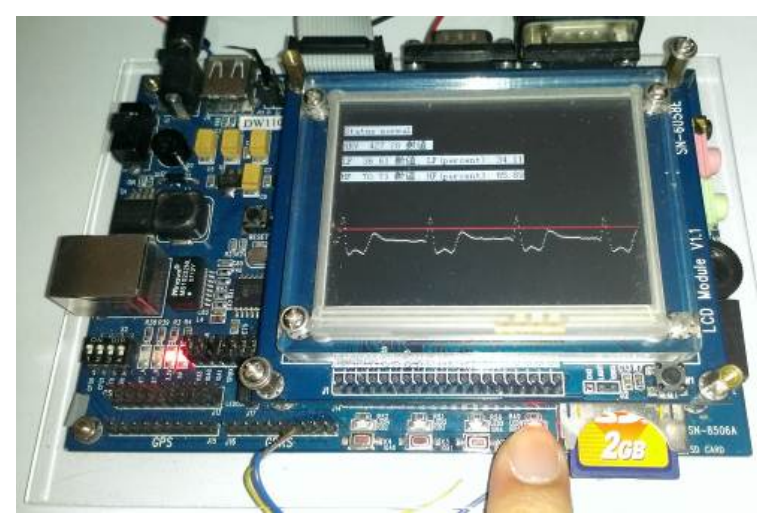

Figure 7. The white ECG signal is shown in the touch panel of the embedded system.

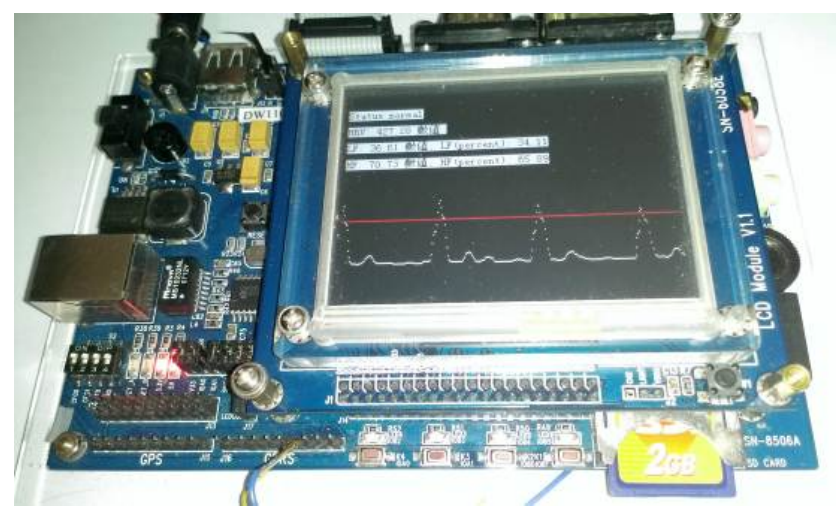

Figure 8. The QRS detection signal is the preprocessed ECG signal after differentiating, squaring and moving-window integration. The red line is the threshold level.

There are three sessions which are 5-minute epochs in the experiment procedure. Session-1 is before LED stimulation, session-2 is during LED stimulation, and session-3 is after LED stimulation. The operating frequency of LED is $10 \mathrm{~Hz}$. The ongoing ECG is recorded in first session and third session. 
Table 2. HRV measurement of one subject

\begin{tabular}{|c|c|c|c|c|c|c|}
\hline Test & $\begin{array}{c}\text { Start } \\
\text { time }\end{array}$ & Epoch & $\begin{array}{c}\text { HRV } \\
(\mathrm{ms})\end{array}$ & $\begin{array}{c}\text { LF } \\
(\%)\end{array}$ & $\begin{array}{c}\text { HF } \\
(\%)\end{array}$ & LF/HF \\
\hline \multirow{2}{*}{ 1st } & $\begin{array}{c}\mathrm{AM} \\
6: 00\end{array}$ & $\begin{array}{c}\text { Session-1 } \\
\text { (Before LED } \\
\text { stimulation) }\end{array}$ & 94.33 & 40.96 & 59.04 & 0.694 \\
\cline { 3 - 7 } 2nd & $\begin{array}{c}\text { Session-3 } \\
\text { (After LED } \\
\text { stimulation) }\end{array}$ & 116.10 & 38.54 & 61.46 & 0.627 \\
\cline { 3 - 7 } & $3: 00$ & $\begin{array}{c}\text { AM } \\
\text { Session-1 } \\
\text { (Before LED } \\
\text { stimulation) }\end{array}$ & 111.56 & 47.74 & 52.26 & 0.914 \\
\cline { 3 - 7 } & $\begin{array}{c}\text { Session-3 } \\
\text { (After LED } \\
\text { stimulation) }\end{array}$ & 97.91 & 33.44 & 66.56 & 0.502 \\
\hline
\end{tabular}

One male subject was tested on twice. The first test was on the morning time AM 6:00 after eight hour sleep. Another test was on the midnight time AM 3:00 after five hour sleep. The measured result was listed in Table-2. In first test, on time-domain analysis, HRV was increased between session-1 and session-3. On frequency-domain analysis, HF (\%) was little increased, and the ratio of LF/HF was little decreased between session-1 and session-3. In second test, on time-domain analysis, HRV was decreased between session-1 and session-3. On frequency-domain analysis, HF (\%) was increased, but the ratio of $\mathrm{LF} / \mathrm{HF}$ was decreased between session-1 and session-3.

\section{Discussion}

HRV is a handy tool for clinical evaluation, one that includes HF as an indicator of parasympathetic activity, LF for the combination of sympathetic and parasympathetic activity, and LF/HF ratio for sympathetic nervous system activity. In this experiment, the subject was required to sit in a chair with opened eyes. LED was radiated at the Neiguan point (PC6) of subject. In first test, HF (\%) and the ratio of LF/HF were little changed between session-1 and session-3. The reason could be the autonomous nervous system had been on balanced status after eight hour sleep, so there was no particular effect on LED stimulation but HRV was increased. In second test, It could the test was hold on midnight and the subject had only five hours sleep, so LF (\%) value was closed to HF (\%). The subject was both sympathetic and parasympathetic dominantly on second test. After LED stimulation, HF (\%) was increased. It could mean that the parasympathetic nervous system had come to be dominant. LED stimulation help to relax the subject and rebalanced the autonomous nervous system. According to $\mathrm{Wu}$ et al. [11] research, low level laser to stimulate on Neiguan point (PC6) can adjust the ANS of the night shift workers.

In the study, we also propose an alert concept. If SDNN or LF were out of the reference range in Table-1, the system will remind the object to turn on LED stimulation to drive ANS balance. But the complete criteria still need to be built with much more data from tests.

\section{Conclusions}

A system with ECG monitoring and low energy LED light stimulation is proposed. In the study, ECG signal is calculated to get time-domain and frequency domain HRV and ANS was analyzed. When the calculated values are out of the self-defined criteria, the system will remind the user to turn on LED to stimulate specific body site (ex. Neiguan point (PC6)) to balance ANS. In additional, the operating frequency and the dosage are adjustable, the default setting is $10 \mathrm{~Hz}$ and the duty cycle is $50 \%$. The data and control parameter can be sent and received between the embedded system and the computer by UART for storage, display, calculation and analysis. In the future, more physiological signals such as electroencephalogram, photoplethysmography etc. would be included into the system for the judgment reference.

\section{Acknowledgments}

We are grateful to the National Science Council of the Republic of China for financial support (contract no.: MOST 104-2221-E-130-005).

\section{REFERENCE}

[1] Conf Proc IEEE Eng Med Biol Soc. 2004;3:2192-5

[2] Carey. Merritt, H. Troy Nagle, Fellow, IEEE, and Edward Grant, Senior, 2009. Fabric-Based Active Electrode Design and Fabrication for Health Monitoring Clothing. IEEE Transactions on Information Technology in Biomedicine, Vol.13, No.2, March 2009.

[3] D Kotecha, G New, M D Flather, D Eccleston, J Pepper, and H Krum, 2012. Five-minute heart rate variability can predict obstructive angiographic coronary disease. Heart 2012 98:395-401.

[4] Salansky, N., Fedotchev, A., and Bondar, A. 1998. Responses of the nervous system to low frequency stimulation and EEG rhythms: clinical implications. Neurosci Biobenav 22 (3), 395-409.

[5] T. Karu, L. Pyatibrat, nad G. Kalendo. 1995. Irradiation with He-Ne laser increases ATP level in cells cultivated in vitro. J. Photochem. Photobiol. B: Biol. 27 (3), 219-223.

[6] Bond, T.J., Lundy, J., 2006. Physical therapy following peripheral nerve surgeries. Clin. Podiatr. Med. Surg. 23 (3), 651-666.

[7] Walker, J., 1983. Relief from chronic pain by low power laser irradiation. Neurosci Lett. 43 (2-3), 339-344.

[8] Paul H Desan, Andrea J Weinstein, Erin E Michalak, Edwin M Tam, Ybe Meesters, Martine J Ruiter, Edward Horn, John Telner, Hani Iskandar, Diane B Boivin and Raymond W Lam. 2007. A controlled trial of the Litebook light-emitting diode (LED) light therapy device for treatment of Seasonal Affective Disorder (SAD). BMC Psychiatry. 
[9] Daniel Barolet. 2008. Light-Emitting Diodes (LEDs) in Dermatology. Seminars in Cutaneous Medicine and Surgical 27:227-238

[10] Natalia Adamskaya a, Peter Dungel a, Rainer Mittermayr, Joachim Hartinger, Georg Feichtinger, Klemens Wassermann, Heinz Redl, and Martijn van Griensven. 2011. Light therapy by blue LED improves wound healing in an excision model in rats. Injury, Int. J. Care Injured 42917 921.

[11] Jih-Huah Wu, Hsin-Yang Chen, Yu-Ju Chang, Hung-Chien Wu, Wen-Dien Chang, Yi-Jing Chu, Joe-Air Jiang. 2009. Study of Autonomic Nervous Activity of Night Shift Workers Treated with Laser Acupuncture. Photo medicine and Laser Surgery. 27, 273-279.

[12] Jih-Huah Wu, Wen-Dien Chang, Chang-Wei Hsieh, Joe-Air Jiang, Wei Fang, Yi-Chia Shan and Yang-Chyuan Chang. 2012. Effect of Low Level Laser Stimulation on EEG. Hindawi Publishing Corporation. Evidence-Based
Complementary and Alternative Medicine. Volume 2012, Article ID 951272, 11 pages.

[13] Jih-Huah Wu, Wei Fang, Yi-Chia Shan, and Yang-Chyuan Chang. Effect of Infrared LED Array Stimulation on EEG. Proceeding (791) Biomedical Engineering, 2013.

[14] Jiapu Pan and Willis J. Tompkins 1985. A Real-Time QRS Detect Algorithm. IEEE TrF Bio-Med Eng, vol.33, BME-32, No.3, pp.230-236.

[15] Berger, R. D., S. Akselrod, D. Gordon and R. J. 1986. Cohen. An efficient algorithm for spectral analysis of heart rate variability. IEEE Trans Bio-Med Eng, vol. 33, no. 9, pp. 900-904.

[16] Task Force of The European Society of Cardiology and The North American Society of Pacing and Electrophysiology, "Heart rate variability Standards of measurement, physiological interpretation, and clinical use". European Heart Journal 17, pp. 354-381, 1996. 